\title{
DESENVOLVIMENTO E AVALIAÇÃO DO PROCESSO DE ACETILAÇÃO CATALÍTICA DA SACAROSE
}

\author{
S. R. T. SILVA ${ }^{1}$, E.B.M. MEDEIROS ${ }^{1}$, C.A.M. ABREU ${ }^{1}$ e N.M. LIMA FILHO ${ }^{1}$ \\ ${ }^{1}$ Universidade Federal de Pernambuco, Departamento de Engenharia Química \\ E-mail para contato: med@ufpb.br
}

\begin{abstract}
RESUMO - Desenvolveu-se o processo de acetilação da sacarose com anidrido acético ativado na presença de catalisadores solúveis, acetato de sódio e hidróxido de sódio, procurando direcionar a reação para formação de octaacetato de sacarose, em reator batelada, agitado mecanicamente e operando com refluxo. Experiências realizadas em intervalos de tempo de 0 a 90 minutos, nas temperaturas de 115,125 e $135^{\circ} \mathrm{C}$ e a pressão atmosférica, apresentaram rendimentos $(\eta)$, definido como porcentagem do produto obtido em relação ao produzido teoricamente, atingindo-se os valores $72,74 \%$, $76,92 \%$ e $99,23 \%$ respectivamente. Objetivando a quantificação do comportamento cinético dos grupos de reações envolvida no processo, apresentou-se hipótese do modelo cinético com base num mecanismo de acetilação da sacarose em presença do acetato de sódio, permitindo verificar um aumento da constante de velocidade da reação em função da temperatura, 0,00474 $\min ^{-1} ; 0,00559 \min ^{-1}$ e 0,00899 $\mathrm{min}^{-1}$ respectivamente.
\end{abstract}

Palavras-chave: Sacarose, Acetilação, Octaacetato de sacarose, Catalisadores homogêneos.

\section{INTRODUÇÃO}

Considerando a variedade funcional dos carboidratos, e em especial da sacarose, observa-se a possibilidade de obtenção de estruturas químicas diversas, por interação com diversos reagentes, os quais podem conduzir a diferentes produtos, permitindo aplicações múltiplas e relevantes. Tendo em vista estes aspectos e tomando como base a disponibilidade dos carboidratos a partir de fontes vegetais agrícolas, a sacarose apresenta-se como matéria-prima com elevado potencial de valorização. Suas funcionalizações químicas podem resultar em produções seletivas, inserindo-se no contexto econômico dos processos industriais.

No âmbito de sua funcionalidade a sacarose reage com ácidos graxos para formar ésteres e poliésteres, os quais possuem inúmeras aplicações (Lu et al., 2004). A reação de acetilação da sacarose utilizando catalisadores homogêneos foi estudada por Silva 2007, com proposição de um modelo cinético de pseudo-segunda ordem para a formação do produto octaacetato de sacarose em relação à concentração do reagente anidrido acético e a partir das considerações dos efeitos da variação do catalisador acetato de sódio.

Le Coent et al., 2003, investigaram a possibilidade de preparação dos ésteres de sacarose sem nenhum solvente orgânico presente na reação. A reação foi realizada em um reator descontínuo agitado mecanicamente, envolvendo mais de uma fase em uma 
reação sólido/sólido/líquido entre a sacarose (sólida) e o metil palmitato (líquido). O segundo sólido (carbonato do potássio) agiu como um catalisador. O meio da reação foi heterogêneo e a viscosidade aumentou com a extensão da reação química.

Estudos relacionados aos ésteres de sacarose, em várias aplicações, têm mostrado boas propriedades funcionais, tais como emulsificação, viscosidade, propriedades reológicas e propriedades interfaciais (Yanke et al., 2004). A introdução da sacarose em polímeros tradicionais (poliacrilatos, poliamidas, polietileno, etc...) pode estender suas aplicações em função da incorporação de grupos polares, formação de copolímeros quirais, além de se tornarem parcialmente biodegradáveis. Poliuretanas produzidas com sacarose vêm sendo apontadas como polímeros com excelentes características a anti-chama (Das et al., 2005).

Considerando a importância dos acetatos de sacarose com as suas possíveis aplicações industriais, o presente trabalho teve como objetivo estudar a síntese do octaacetato de sacarose utilizando catalisadores homogêneos de acetato de sódio e hidróxido de sódio; avaliando a influência de parâmetros operacionais sobre o processo de síntese e desenvolvimento de modelagem de descrição do comportamento cinético do processo de acetilação.

\section{MATERIAIS E MÉTODOS}

As experiências de processamento da sacarose com anidrido acético em presença do catalisador acetato de sódio foram executadas de duas maneiras: no primeiro caso, um processo de mistura dos dois reagentes foi realizado em presença do catalisador com elevação simultânea da temperatura, enquanto no segundo modo operatório realizou-se uma ativação prévia do anidrido acético em presença do catalisador com adição posterior da sacarose. Estes dois métodos são detalhados a seguir:

\subsection{Método Experimental - 1}

São pesados $3,75 \pm 0,02 \mathrm{~g}$ de catalisador acetato de sódio e misturados a $100 \pm 0,1$ $\mathrm{ml}$ de anidrido acético num balão volumétrico de 0,5 litros de capacidade compondo o reator de batelada de mistura representado na Figura 1.

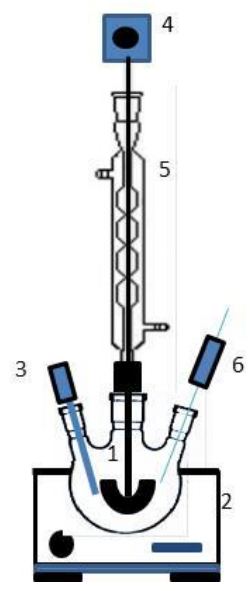

1. Reator de Vidro

2. Manta Aquecedora

3. Termopar

4. Agitador Mecânico

5. Condensador de Bola

6. Coletor de Amostra

Figura 1 - Reator batelada de mistura para acetilação de carboidratos. 
Em seguida, adiciona-se 37,5 $\pm 0,02 \mathrm{~g}$ de sacarose e inicia-se o aquecimento com agitação até atingir a temperatura de $125^{\circ} \mathrm{C}$, dando-se inicio a contagem dos tempos de operação, mantendo-se a temperatura de $125^{\circ} \mathrm{C}$, a pressão atmosférica.

Ao final do tempo de operação, cessado o aquecimento e a agitação, mistura-se o conteúdo do produto de reação, com água a $0^{\circ} \mathrm{C}$ deixando-se a mistura em repouso por cerca de 30 minutos. O produto oleoso que se separa é filtrado e lavado com água fria, para em seguida ser recristalizado com álcool etílico PA até que medidas sucessivas de pontos de fusão apresentem valores constantes. Após duas recristalizações obtém-se o produto com ponto de fusão igual a $85 \pm 1^{\circ} \mathrm{C}$.

\subsection{Método Experimental - 2}

Procede-se de modo semelhante ao do método experimental-1 quanto à mistura de anidrido acético e catalisador. Deixa-se esta mistura ser aquecida até temperatura de reação sob agitação constante, quando então adiciona-se lentamente a sacarose pura eliminando-se temporariamente o aquecimento, mantendo-se em seguida a temperatura a $135^{\circ} \mathrm{C}$ por 15 minutos. No final dos 15 minutos destila-se o excesso de ácido acético formado durante a reação e dissolve-se o resíduo obtido em álcool etílico, filtrando-o em seguida em contato com 8 gramas de carvão ativado à quente. Após a retirada do ácido acético, procede-se com a recristalização em álcool etílico puro até que o ponto de fusão seja constante e igual a $85 \pm 1^{\circ} \mathrm{C}$.

Para a identificação dos acetatos de carboidratos foram realizadas análises por Cromatografia em Camada Delgada (CCD) e Espectrofotometria de Infravermelho IFS. Além da Cromatografia Líquida de Alta Eficiência - CLEA com detecção por índice de refração e coluna NH2-nucleosil, sem êxito na separação, devido às proximidades de suas polaridades e afinidades pelos sistemas de fases móveis testados.

As análises por CCD para os produtos da reação seguidos pelos métodos de execução do experimento 1 e 2 indicaram que o octaacetato de sacarose sintetizado era constituído principalmente de material hidrofóbico. O produto antes da purificação com álcool etílico continha traços de pentaacetato de glicose e tetraacetato de frutose, após duas recristalizações com álcool etílico, o grau de pureza foi verificado a partir do ponto de fusão com resultado igual a $85 \pm 1^{\circ} \mathrm{C}$.

\section{RESULTADOS E DISCUSSÃO}

\subsection{Caracterização dos ésteres de carboidratos}

As experiências de acetilação da sacarose foram inicialmente realizadas a níveis de testes com anidrido acético em presença do catalisador de acetato de sódio. Procedendo inicialmente com o pré-aquecimento do anidrido acético em presença do catalisador a $135^{\circ} \mathrm{C}$ com uma posterior adição da sacarose, conforme foi descrito no método experimental-2, no intuito da diminuição dos efeitos de hidrólise da sacarose, para formação de pentaacetato de glicose e tetraacetato de frutose.

As análises realizadas por espectrofotometria de infravermelho (IFS 66 Bruker) permitiram a caracterização dos grupos funcionais. Nas Figuras 2 e 3 são 
observados os sinais correspondentes à deformação axial da ligação $\mathrm{C}-\mathrm{H}$ dos grupos metil e metileno da cadeia de ácidos graxos para o octaacetato de sacarose e sacarose.

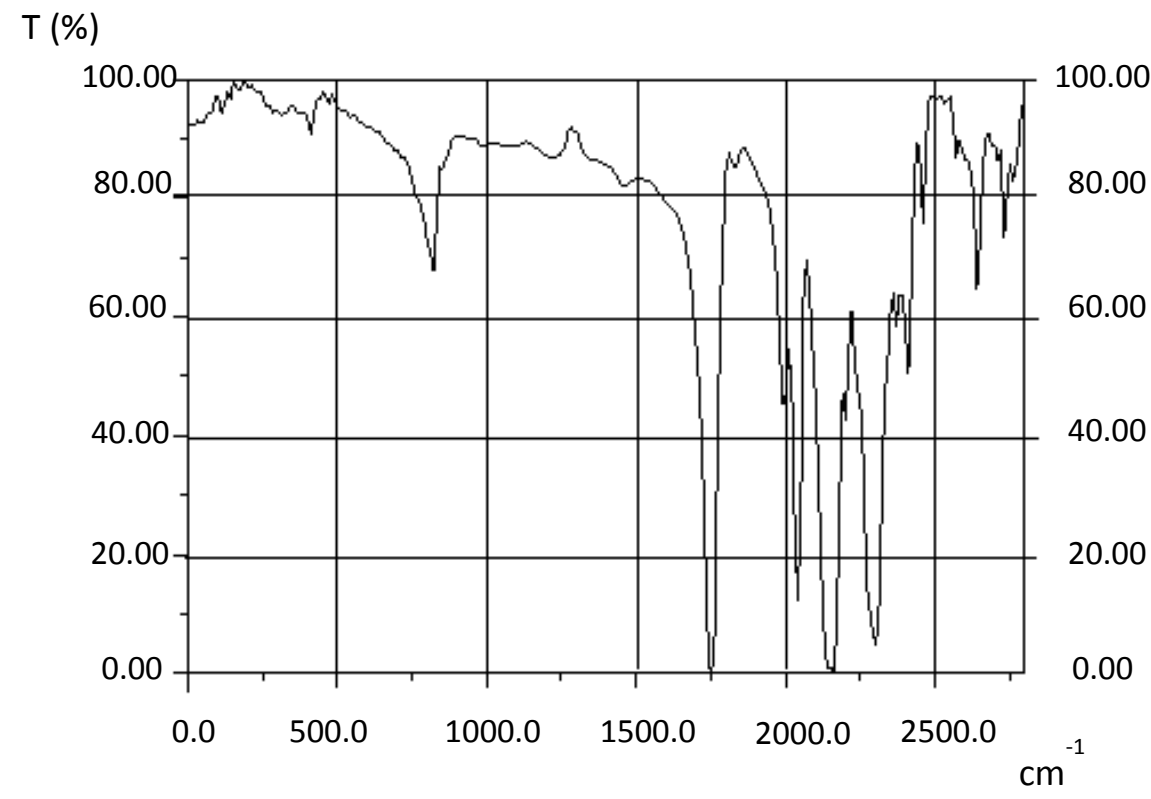

Figura 2 - Espectro no Infravermelho do octaacetato de sacarose

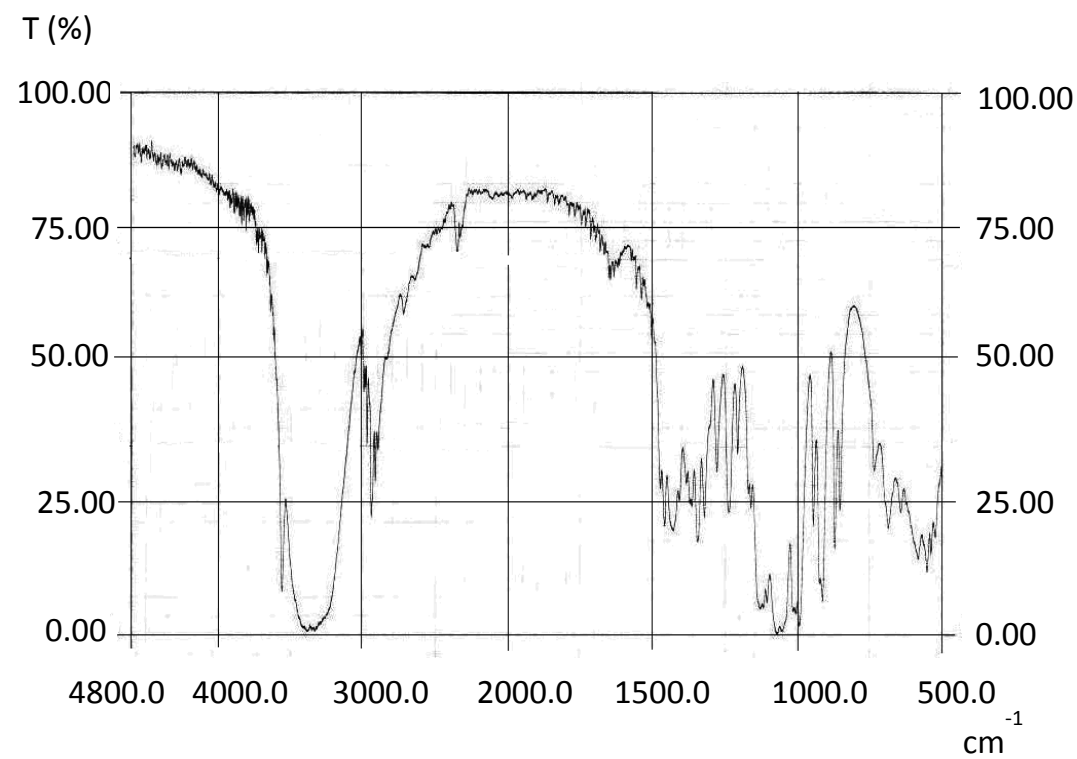

Figura 3 - Espectro no Infravermelho da sacarose

A banda de absorção de hidroxila em $3335 \mathrm{~cm}^{-1}$ que foi observada no espectro da sacarose não foi observada no espectro do octaacetato de sacarose, isto indica que todos os grupos hidroxilas da sacarose foram acetilados na presença do anidrido acético ativado pelo catalisador acetato de sódio.

Uma análise mais minuciosa dos dois espectros de infravermelho permite verificar uma grande similaridade entre os mesmos, que apresentam sinais de absorção dos grupos característicos para esses tipos de compostos. Em todos os espectros 
observa-se um sinal fraco centrado aproximadamente em $750 \mathrm{~cm}^{-1}$, o qual pode ser atribuído aos estiramentos simétricos e assimétricos dos grupos metilas terminais ($\left.\mathrm{CH}_{3}\right)$ dos grupos metilênicos $\left(-\mathrm{CH}_{2}\right)$ e dos grupos metinicos $(-\mathrm{CH})$ presente no anel. Na região compreendida entre $1750 \mathrm{~cm}^{-1}$, verifica-se uma banda forte e aguda, característica do grupamento carbonila, evidenciando-se a presença deste na molécula.

Já na região em aproximadamente $2000 \mathrm{~cm}^{-1}$ para octaacetato de sacarose, notase claramente a presença de duas bandas fortes que podem ser atribuídas às vibrações $C$ $\mathrm{O}$ do composto. $\mathrm{Na}$ verdade, estas bandas são características de vibrações simétricas de ésteres, que podem ser acoplados ou não: $\mathrm{C}-\mathrm{C}(=\mathrm{O})-\mathrm{O}$ e $\mathrm{O}-\mathrm{C}-\mathrm{C}$, sendo o pico um pouco maior o mais importante.

E a partir da comparação do espectro da Figura 2 com o da Figura 3 observa-se a ausência da banda correspondente aos estiramentos simétricos e assimétricos do grupo hidroxila (-OH), situado no intervalo $3700-3100 \mathrm{~cm}^{-1}$ na Figura 3. E na Figura 2 observa-se uma banda forte, compreendida em $1750 \mathrm{~cm}^{-1}$ característica da deformação axial do grupo carbonila $(-\mathrm{C}=\mathrm{O})$. Com base nesta comparação, infere-se que todo o composto foi acetilado, obtendo-se o produto desejado.

\subsection{Acetilação Catalítica da Sacarose}

Na Figura 4 são mostrados os resultados dos rendimentos em octaacetato de sacarose $(\eta)$, definido como porcentagem do produto obtido em relação ao produzido teoricamente. Abordando as particularidades relativas às atividades dos dois catalisadores testados. Em razão da necessidade da validação de uma hipótese de modelo fenomenológico, que leva em consideração a dependência da formação e/ou da presença do acetato de sódio para ativação do anidrido acético na reação de acetilação da sacarose. Tomando-se " $\mathrm{t}_{0}$ " como o tempo a partir do momento que a reação atingir a temperatura isotérmica de processamento em refluxo a pressão atmosférica.

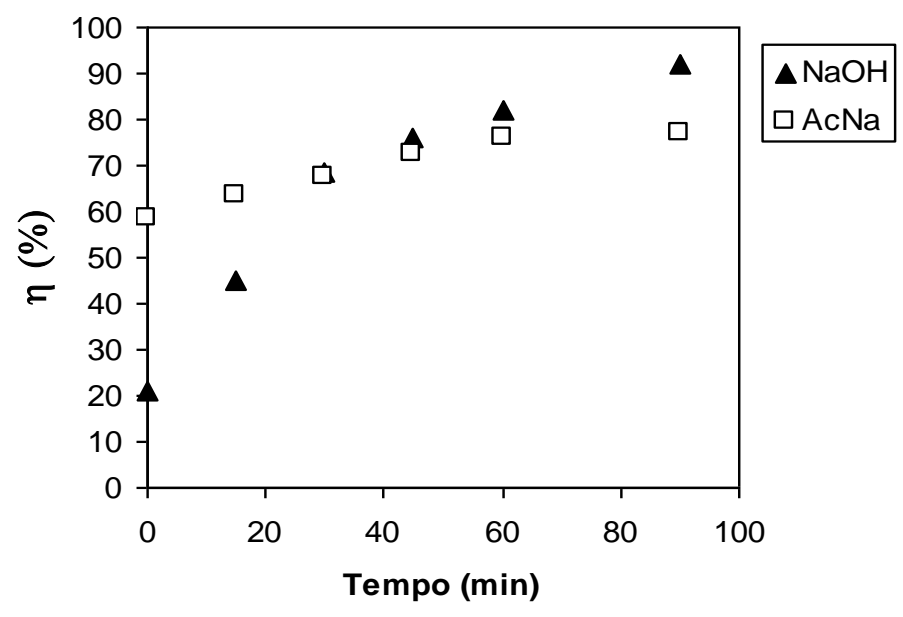

Figura 4 - Influência da Natureza do Catalisador no Rendimento. Condições Operatórias: $\mathrm{T}=125^{\circ} \mathrm{C},[\mathrm{AcNa}]_{0}=[\mathrm{NaOH}]_{0}=0,18 \mathrm{~mol} / \mathrm{L},[\text { Anidrido }]_{0} /[\mathrm{SuOH}]_{0}=1,207$.

Onde $[\mathrm{AcNa}]_{0}=$ concentração inicial de acetato de sódio, $[\mathrm{NaOH}]_{0}=$ concentração inicial de $\mathrm{NaOH}$, [Anidrido $]_{0}=$ concentração inicial de anidrido acético e $[\mathrm{SuOH}]_{0}=$ concentração inicial de sacarose. 
Observa-se, na Figura 4, a evolução dos rendimentos em octaacetato de sacarose de forma diferente para os dois catalisadores testados, validando a hipótese de que o uso do hidróxido de sódio como catalisador deve levar em conta primeiramente a formação do ácido acético que, em presença do hidróxido de sódio, forma o acetato de sódio, que é responsável pela catálise da reação ao longo do tempo, confirmando a possibilidade da influência efetiva do acetato de sódio como catalisador para a reação de acetilação.

\subsection{Modelagem Cinética da Acetilação}

Levando-se em conta a hipótese do catalisador acetato de sódio realizar de forma efetiva a ativação do anidrido acético, postulou-se uma proposta de modelo fenomenológico baseado na constatação dos resultados experimentais em relação à formação de intermediários reacionais instáveis: $\mathrm{CH}_{3} \mathrm{C}^{*} \mathrm{O},\left(\mathrm{CH}_{3} \mathrm{COO}\right)_{2} \mathrm{Na}^{*}$. Estes complexos formados entre o anidrido acético e o catalisador (acetato de sódio), permitindo a interação com a sacarose $(\mathrm{SuOH})$ são apresentados nas etapas I, II e III.

$$
\begin{aligned}
& \left(\mathrm{CH}_{3} \mathrm{CO}\right)_{2} \mathrm{O}+\mathrm{CH}_{3} \mathrm{COONa} \rightleftarrows \mathrm{CH}_{3} \mathrm{C}^{*} \mathrm{O}+\left(\mathrm{CH}_{3} \mathrm{COO}\right)_{2} \mathrm{Na}^{*} \\
& \mathrm{SuOH}+\mathrm{CH}_{3} \mathrm{C}^{*} \mathrm{O} \rightarrow \mathrm{Su}^{*}+\mathrm{CH}_{3} \mathrm{COOH} \\
& \mathrm{Su}^{*}+\left(\mathrm{CH}_{3} \mathrm{COO}\right)_{2} \mathrm{Na}^{*} \rightarrow \mathrm{OAcSu}+\mathrm{CH}_{3} \mathrm{COONa}
\end{aligned}
$$

$\mathrm{O}$ balanço de massa do octaacetato de sacarose (OAcSu), incluindo lei cinética de primeira ordem para os componentes envolvidos, permite formular uma equação do modelo (equação 1).

$$
\frac{d[\mathrm{OAcSu}]}{d t}=k_{3}\left[\mathrm{Su}^{*}\right]\left[\left(\mathrm{CH}_{3} \mathrm{COO}\right)_{2} \mathrm{Na}^{*}\right]
$$

Onde $\mathrm{k}_{3}$ é a constante cinética da reação III. Aplicando a teoria do estado estacionário para os componentes intermediários instáveis, segundo o mecanismo fenomenológico de reação, $\mathrm{Su}^{*}$ e $\mathrm{CH}_{3} \mathrm{C}^{*} \mathrm{O}$, têm-se as suas taxas de reação:

$$
\begin{aligned}
& \frac{d\left[\mathrm{Su}^{*}\right]}{d t}=k_{2}[\mathrm{SuOH}]\left[\left(\mathrm{CH}_{3} \mathrm{C}^{*} \mathrm{O}\right)\right]-k_{3}\left[\mathrm{Su}^{*}\right]\left[\left(\mathrm{CH}_{3} \mathrm{COO}\right)_{2} \mathrm{Na}^{*}\right] \simeq 0 \\
& \begin{aligned}
\frac{d\left[\mathrm{CH}_{3} \mathrm{C}^{*} \mathrm{O}\right]}{d t}= & k_{1}\left[\left(\mathrm{CH}_{3} \mathrm{CO}\right)_{2} \mathrm{O}\right]\left[\mathrm{CH}_{3} \mathrm{COONa}\right] \\
& \left.-k_{-1}\left[\mathrm{CH}_{3} \mathrm{C}^{*} \mathrm{O}\right]\left[\mathrm{CH}_{3} \mathrm{COO}\right)_{2} \mathrm{Na}^{*}\right]-k_{2}[\mathrm{SuOH}]\left[\left(\mathrm{CH}_{3} \mathrm{C}^{*} \mathrm{O}\right] \simeq 0\right.
\end{aligned}
\end{aligned}
$$

Substituindo as expressões (2) e (3) na expressão (1), obtém-se:

$\frac{d[\mathrm{OAcSu}]}{d t}=\frac{k_{2} k_{1}\left[\left(\mathrm{CH}_{3} \mathrm{CO}\right)_{2} \mathrm{O}\right]\left[\mathrm{CH}_{3} \mathrm{COONa}\right][\mathrm{SuOH}]}{\left.k_{-1}\left[\mathrm{CH}_{3} \mathrm{COO}\right)_{2} \mathrm{Na}^{*}\right]+k_{2}[\mathrm{SuOH}]}$ 
E a partir de hipótese postulada pelas constatações experimentais em relação ao catalisador, tem-se que:

$\left.k_{-1}\left[\mathrm{CH}_{3} \mathrm{COO}\right)_{2} \mathrm{Na}^{*}\right] \ll k_{2}[\mathrm{SuOH}]$; então $\left.k_{-1}\left[\mathrm{CH}_{3} \mathrm{COO}\right)_{2} \mathrm{Na}^{*}\right]+k_{2}[\mathrm{SuOH}]$ pode ser substituida por $k_{2}[\mathrm{SuOH}]$, obtendo-se:

$\frac{d[\mathrm{OACSu}]}{d t}=k_{1}\left[\mathrm{CH}_{3} \mathrm{COONa}\right]\left[\left(\mathrm{CH}_{3} \mathrm{CO}\right)_{2} \mathrm{O}\right]$

Considerando a regeneração constante do catalisador que participa efetivamente do mecanismo de reação, tem-se:

$\frac{d[\mathrm{OAcSu}]}{d t}=k\left[\left(\mathrm{CH}_{3} \mathrm{CO}\right)_{2} \mathrm{O}\right]$

Como o monitoramento foi realizado pela produção do octaacetato de sacarose, tem-se a partir do avanço da reação que $\left[\left(\mathrm{CH}_{3} \mathrm{CO}\right)_{2} \mathrm{O}\right]=\left[\left(\mathrm{CH}_{3} \mathrm{CO}\right)_{2} \mathrm{O}\right]_{0}-[\mathrm{OAcSu}]$, logo:

$\frac{d[\mathrm{OAcSu}]}{d t}=k\left(\left[\left(\mathrm{CH}_{3} \mathrm{CO}\right)_{2} \mathrm{O}\right]_{0}-[\mathrm{OAcSu}]\right)$

A equação diferencial é resolvido numericamente por um método de integração do tipo Runge Kutta de $4^{a}$ ordem, seguido da aplicação de um método de otimização BOX, 2006, procurando-se minimizar a função objetivo $\left(f_{0}\right)$, definida como a diferença quadrática, entre os valores experimentais e calculados das concentrações dos componentes da reação. Comparações entre os valores teóricos e experimentais para a formação do octaacetato de sacarose, nas temperaturas consideradas é mostrada na Figura 5, indicando bom ajuste do modelo proposto quantificando-se desvios médios da ordem de $10^{-2}$.

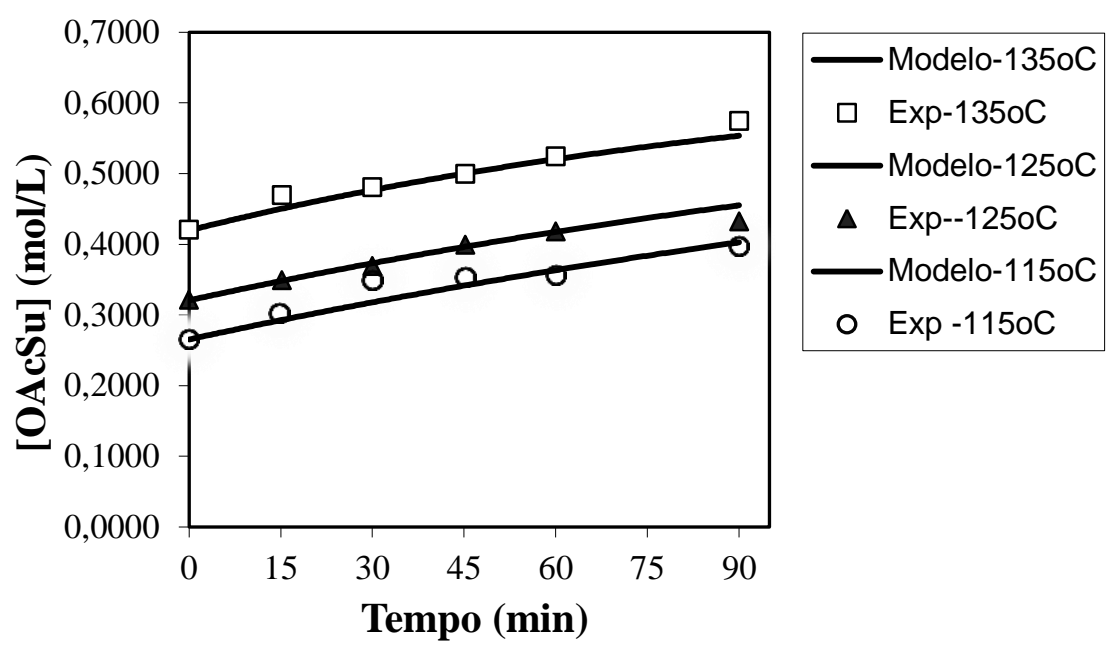

Figura 5 - Comparação entre as concentrações experimentais e calculadas a partir do modelo cinético da produção do octaacetato de sacarose. $\left[\left(\mathrm{CH}_{3} \mathrm{CO}\right)_{2} \mathrm{O}\right]_{0} /[\mathrm{SuOH}]_{0}=1,207$, $[\mathrm{NaAc}]=[\mathrm{NaOH}]=0,18 \mathrm{~mol} / \mathrm{L}, \mathrm{P}=1 \mathrm{~atm}$. 
Os valores da constante de velocidade (k) são mostrados na Tabela 1. Da lei de Arrhenius $k=k_{0} \operatorname{Exp}\left(-E_{a} / R T\right)$, onde é $\mathrm{k}_{0}$ fator de frequência, $\mathrm{R}$ constante dos gases e $\mathrm{T}$ temperatura, consegue-se um ajuste linear, resultando em uma energia de ativação $\left(E_{a}\right)$ de 201,11 cal, valor esse semelhante aos obtidos na literatura.

Tabela 1 - Constantes de Velocidade da Reação de Acetilação da Sacarose na Presença de Acetato de Sódio.

\begin{tabular}{c|c}
\hline Temperatura $\left({ }^{\circ} \mathrm{C}\right)$ & $\mathrm{k}\left(\mathrm{min}^{-1}\right)$ \\
\hline \hline 115 & 0,00474 \\
\hline 125 & 0,00559 \\
\hline 135 & 0,00899 \\
\hline
\end{tabular}

\section{CONCLUSÕES}

A caracterização de octaacetato de sacarose deixa de ser um desafio analítico com a utilização de técnicas aplicadas de ponto de fusão, espectrofotometria de infravermelho, CCD e CLEA na identificação do produto final.

A influência da natureza do catalisador é verificada tal que o acetato de sódio catalisa a reação de acetilação da sacarose com rendimento ( $\eta$ ), de cerca $76 \%$ em octaacetato de sacarose, resultado esse inferior quando comparado ao catalisador de hidróxido de sódio, em torno de $92 \%$, para as condições operatórias estudadas ao final dos 90 minutos de reação em relação à quantidade teórica produzida.

A reação de acetilação da sacarose em presença do catalisador de acetato de sódio descreve bem a hipótese do modelo cinético fenomenológico. Os resultados obtidos a partir da otimização nas três temperaturas utilizadas 115,125 e $135{ }^{\circ} \mathrm{C}$ demonstram que o comportamento cinético é favorável, com um bom ajuste linear, erro da função objetivo em torno $10^{-2}$.

\section{REFERÊNCIAS BIBLIOGRÁFICAS}

DAS, S. K.; REDDY, K. A.; KROVVIDI, V. L. N. R.; MUKKANTI, K. - $\mathrm{InCl}_{3}$ as a powerful catalyst for the acetylation of carbohydrate alcohols under microwave irradiation - Carbohydrate Research 340, 1387-1392, 2005.

KUO-CHENG, LU; HSIEH, S.-Y.; PATKAR, L. N.; CHEN, C. T.; LIN, C. C. - Simple and efficient per-O-acetylation of carbohydrates by lithium perchlorate catalyst Tetrahedron 60, 8967-8973, 2004.

LE COENT, A. -L.; TAYAKOUT,-F.M.; COUENNE, F.; BRIANÇON, S.; LIETO, J.; FITREMANN, -G. J.; QUENEAU, Y.; BOUCHU, A. Kinetic parameter estimation and modelling of sucrose esters synthesis without solvent - Chemical Engineering Science 58, $367-376,2003$.

SILVA, S. R. T., Acetilação Catalítica de Sacarídeos. Influência de Parâmetros Cinéticos - Dissertação de Mestrado, PPEQ - UFPE, 2007.

YANKE, L.; SHUFEN, Z.; JINZONG, QINGHUI, W. Relationship of solubility parameters to interfacial properties of sucrose esters - Colloids and Surfaces A: Physicochem. Eng. Aspects, 248, 127-133, 2004. 